Z. Phys. Chem. 217 (2003) 1207-1219

(C) by Oldenbourg Wissenschaftsverlag, München

\title{
Katalyse: Vom Stein der Weisen zu Wilhelm Ostwald
}

\author{
Von Gerhard Ertl* und Tanja Gloyna \\ Fritz-Haber-Institut der Max-Planck-Gesellschaft, Faradayweg 4-6, D-14195 Berlin
}

(Received July 4, 2003; accepted July 7, 2003)

\section{History of Science / Catalysis}

Ausgehend von Beobachtungen durch Döbereiner und zahlreiche andere führte Berzelius 1835 den Begriff Katalyse ein, deren Wirkung häufig mit dem ,Stein der Weisen “ der mittelalterlichen Alchemie verglichen wird. Erst gegen Ende des 19. Jahrhunderts erfolgte durch Ostwald eine klare Definition auf der Grundlage der Reaktionskinetik. Schließlich wird der heutige Stand der Erkenntnis kurz an einem Beispiel erläutert.

Am 29. Juli 1823 berichtete der damalige Professor für Chemie an der Universität Jena, Johann Wolfgang Döbereiner, an seinen Minister - und dies war niemand geringerer als Goethe - wie folgt: „Ich erlaube mir, Eurer Exzellenz von einer Entdeckung Nachricht zu geben, welche [...] im hohen Maße wichtig erscheint. Ich finde [...], dass das rein metallische staubfeine Platin die höchst merkwürdige Eigenschaft hat, das Wasserstoffgas durch bloße Berührung [...] zu bestimmen, dass es sich mit Sauerstoffgas zu Wasser verbindet, wobei eine bis zum Entglühen des Platins gesteigerte Summe von Wärme erregt wird“ [1]. Die Knallgasreaktion sowie andere Beobachtungen von Platinpräparaten und deren Beeinflussung chemischer Reaktionen hatten Döbereiner zur Annahme geführt, dass die Wirkung ,durch bloße Berührung“ schon bei geringer Menge entstünde, wobei die jeweiligen Platinpräparate selbst ,keine Veränderung“ erlitten und wiederholt verwendet werden könnten, „um neue, vielleicht unendliche Quantitäten“ zu beeinflussen [2]. Etwa seit Beginn des 19. Jahrhunderts waren aus verschiedenen Laboratorien weitere Berichte über die Wirkung vermeintlich selbst unbeteiligter Substanzen auf chemische Reaktionen erschienen, und 1834 faßte Eilhard Mitscherlich die Beobachtungen mit dem Ausdruck ,Zersetzung und Verbindung durch Kontakt“ zusammen [3]. Die Bezeichnung „Katalyse“ für diese eigentümliche Klasse chemischer Er-

* Corresponding author. E-mail: ertl@fhi-berlin.mpg.de 
scheinungen wurde dann 1835 von dem Sekretär der schwedischen Akademie der Wissenschaften, Jöns Jacob Berzelius, eingeführt.

Dass durch Hinzufügung einer kleinsten Stoffmenge andere in ihrer Erscheinung stark beeinflusst werden können, war seit der Antike bekannt, und zwar aus der Wirkung von Hefe, die eine große Teigmenge verwandelt. Dieses ,fermentum“ wurde in der Alchemie mit der Wirkungskraft des Steins der Weisen verglichen, der, in geringster Menge auf ein unedles Metall geworfen, dieses in Gold verwandeln sollte. Ursprünglich ist der Wandel, die Transmutation, nach dem Kriterium der Farbveränderung einer Substanz beurteilt worden. Auch in diesem Zusammenhang war ein vertrautes Phänomen der frühen Praxis, dass eine geringe Menge Farbstoffs große Mengen färben kann. Die Vorstellung von der Fermentwirkung des Steins der Weisen wurde durch alchemistische Traktate des Mittelalters vermittelt und blieb durch eine entsprechende Textsammlung, das Deutsche Theatrum Chemicum (1723 ff.), bis ins 19. Jahrhundert präsent.

Noch der Chemiehistoriker und Liebig-Schüler Hermann Kopp hielt fest, dass nach dieser Lehre der Stein der Weisen ,bei der Projection auf unedles Metall“, d.h. mit diesem „,in Berührung gebracht“, „,eine unverhältnismässig grosse Menge - nach der Versicherung alchemistischer Autoritäten des vieltausend- und selbst vielbillionenfache Gewicht in bestes Gold umzuwandeln vermöge“ [4]. Mit dieser Vorstellung der Unverhältnismäßigkeit war die assoziative Verknüpfung „Stein der Weisen“- „Katalysator“ vorbereitet. Gleichwohl betonte schon Kopp den wesentlichen Unterschied beider, da allein ,der Stein der Weisen - die zu einer Transmutation verwendete Quantität desselben nämlich - (...) nach der Ausübung seiner metallveredlenden Wirksamkeit nicht mehr (existirt)“ [5]. Die Verbindung Stein der Weisen - Katalysator hatte allerdings auch nahegelegen, weil der Vorgang der biologischen Fermentwirkung insbesondere seit den Arbeiten Justus Liebigs häufig Gegenstand der Beobachtungen zu Katalyse war.

Heute, nach Wilhelm Ostwalds Definition von Katalyse, ist es nicht nur üblich, umgangssprachlich das, was einen Prozeß beschleunigt, als „Katalysator" zu bezeichnen, sei es eine Person, ein Ding oder ein Sachverhalt, sondern es wird vor allem in der Literatur zur Alchemie die Assoziation Stein der Weisen - Katalysator in diesem Sinn gebraucht: „Von der Annahme, die Metalle würden im Inneren der Erde einen langsamen Reifungsprozeß durchmachen, der beim Blei beginne und beim Gold ende, leitet sich auch die Vorstellung $a b$ “, der Stein der Weisen ,sei ein Beschleunigungsmittel dieses natürlichen Vorgangs, modern ausgedrückt: ein Katalysator. Ein an sich natürlicher, aber sehr langsamer Vorgang werde ... beschleunigt" [6]. Die Vorstellung, mit der Zugabe des Steins der Weisen als Ferment würde, analog zur Behandlung von Pflanzensamen, die natürliche Entwicklung angenommener Metallsamen soweit optimiert, dass sich schließlich sogar einer des Bleis in den von Gold verwandle und sich dann entfalte, geht auf arabische Alchemisten zurück. Deren mittelalterliche und frühneuzeitliche christliche 
Ausdeutung hielten wiederum die Texte des genannten Theatrum Chemicum wach.

Ostwald selbst äußerte sich Jahre nach seiner Definition zum historischen und sachlichen Zusammenhang von Alchemie und Chemie wie folgt: „Unter den ersten Träumen, welche das beginnende wissenschaftlich-technische Denken erfüllten, spielt das große Elixier eine sehr hervorragende Rolle. Das Suchen nach dem Stein der Weisen oder der Tinktur, die aus unedeln Metallen Gold zu machen und dem menschlichen Körper dauernde Gesundheit zu verleihen vermochte, ist eine Hauptquelle der Chemie gewesen, dieser Wissenschaft, in der sich nicht weniger deutlich wie in anderen darstellt, wie viel von solchen Träumen erreichbar ist, da es tatsächlich erreicht worden ist. Das große Elixier ist heute zwar noch nicht restlos, aber doch zu einem sehr großen Teile gefunden. Zwar vermag man noch nicht gerade Blei in Gold zu verwandeln. Es sind aber dafür zahllose andere Verwandlungen von geringwertigem Material in wertvollstes entdeckt worden, so dass jenes Problem, das ohnehin heute mehr symbolisch anmutet, ganz in den Hintergrund getreten ist. (...) Alle diese Verwirklichungen alter Menschheitsträume sind auf einem einzigen Wege gewonnen worden. Dieser Weg heißt Wissenschaft" [7]. Unter der Betonung des Fortschritts der Wissenschaft hatte sich zuvor Justus Liebig sogar zu der Aussage verstiegen: „Die Alchemie ist niemals etwas anderes als die Chemie gewesen; (...) Der Stein der Weisen ist in seiner Vollkommenheit nichts anderes gewesen als die Wissenschaft der Chemie“" [8].

Beide Einschätzungen setzten freilich die Entwicklung einer strengen $\mathrm{Be}$ grifflichkeit innerhalb dieser Wissenschaft voraus; daher zurück zu den Anfängen der Katalyse-Forschung: Experimentelle Befunde, die auf das Phänomen hinwiesen, hatte Berzelius in seinen seit 1820 verfassten „Jahres-Berichten“ immer wieder aufgeführt, aber erst im fünfzehnten Band brachte er die spezifische Wirkungsweise durch Namensgebung auf einen Punkt: „Ich werde sie daher, um mich einer in der Chemie wohlbekannten Ableitung zu bedienen, die katalytische Kraft der Körper, und die Zersetzung durch dieselbe Katalyse nennen, gleichwie wir mit dem Wort Analyse die Trennung der Bestandtheile der Körper, vermöge der gewöhnlichen chemischen Verwandtschaft, verstehen“ [9]. Die Möglichkeit von Zwischenverbindungen, an denen ein Katalysator beteiligt ist, schloß Berzelius nicht aus; so formulierte er im Sinn der Lehre von den chemischen Verwandtschaften, dass entsprechende Stoffe ,in dem Körper“, auf den sie wirkten, ,eine Umsetzung der Bestandtheile in anderen (!) Verhältnissen (!)“ erzeugten, ,ohne daß sie dabei mit ihren Bestandtheilen nothwendig selbst Theil nehmen, wenn dies auch mitunter der Fall sein kann" [10].

Im Unterschied zur Analyse vermutete Berzelius für die katalytische Kraft, „daß Körper durch ihre bloße Gegenwart, und nicht durch ihre Verwandschaft, die bei dieser Temperatur schlummernden Verwandtschaften zu erwecken vermögen, so daß zufolge derselben in einem zusammengesetzten Körper die Elemente sich in solchen anderen Verhältnissen ordnen“ [11]. Daher äußerte er 
sich in einem Brief vom 10. April 1835 an Justus Liebig auch wie folgt: „Ich nenne die neue Kraft (mag sie sein was sie will) katalytische Kraft der Körper und das Zerlegen durch katalytische Kraft, z. B. das des Zuckers durch Wasser und Hefe, Katalysis, was mir gut zu passen scheint, da es ein Pendant zu Analysis ist, welche durch Wahlverwandtschaft ausgeführt wird" [12].

Der Stellenwert, den die katalytische Kraft bereits für Berzelius innehatte, erschließt sich in vollem Umfang angesichts ihres Wirkungskreises: Er bestimmte diese „,neue Kraft“ als „eine eben sowohl der unorganischen, als der organischen Natur angehörige“; ja er nahm an, dass sie „gewiss mehr, als man bis jetzt dachte, verbreitet“ sein dürfte [13]. So war für die „chemischen Prozesse(n) in der lebenden Natur“ zu vermuten, „daß in den lebenden Pflanzen und Thieren tausende von katalytischen Prozessen ... vor sich gehen, und die Menge ungleichartiger chemischer Zusammensetzungen hervorbringen, von deren Bildung aus dem gemeinschaftlichen rohen Material, dem Pflanzensaft oder dem Blut, wir nie eine annehmbare Ursache einsehen konnten, die wir künftig vielleicht in der katalytischen Kraft des organischen Gewebes, woraus die Organe des lebenden Körpers bestehen, entdecken werden“ [14].

Diese Übertragung katalytischer Prozesse auf den Funktionszusammenhang von Organismen macht deutlich, in welcher Linie Berzelius' Begriff der katalytischen Kraft stand: Die „Ursache“ für den genannten Vorgang der Umwandlung und Anverwandlung ,chemischer Zusammensetzungen“, welche für die Bildung und Erhaltung lebender Körper notwendig sind, wurde bis dahin in einer besonderen vis vitalis gesucht. Die Ablösung der „Lebenskraft“ durch die katalytische Kraft brachte P. Walden späterhin so zum Ausdruck: „Die vis vitalis verschwand nicht, ohne vorher eine andere vis occulta, die katalytische Kraft, gleichsam als Stellvertreterin im lebenden Organismus geschaut zu haben“ [15], und auch Berzelius sprach von der katalytischen Kraft als einer vis occulta [16]. Alles in allem blieb seine anfängliche Rede von der Entdeckung einer „,neuen Kraft“, die u. a. das Aufgabenfeld der Lebenskraft übernehmen sollte, uneindeutig. Mit Sicherheit festzuhalten ist für Berzelius' Verständnis, dass diese Kraft - „mag sie sein was sie will“ - im „Zerlegen“ chemischer Zusammensetzungen durch „Katalysis“ sowohl begrifflich als auch sachlich das „Pendant zu Analysis“ bildete. Und als Ergänzung zur chemischen „Wahlverwandtschaft“ war sie Ausdruck für eine neu gewonnene Erkenntnis über das Zusammenspiel von Körpern und ihren chemischen Bestandteilen.

In der Folgezeit wurde der Stellenwert des von Berzelius benannten Phänomens im allgemeinen - positiv wie negativ - nicht weiter in Frage gestellt. Hingegen boten die Uneindeutigkeit des Kraftbegriffs, der sich zudem mit dem Begriffsfeld der vis vitalis überschnitt, sowie der Mangel einer theoretischen Erklärung Anlaß zu Kritik und Weiterentwicklung. Mit Blick auf die vermeintliche „Eigenständigkeit“ katalytischer Kraft sind gleichermaßen Widerspruch und Bestätigung festzustellen: Bestes Bespiel für ersteres ist Justus Liebig und für letzteres Julius Robert Mayer. 
Anhand des Briefwechsels zwischen Liebig und Friedrich Wöhler, der Berzelius' Jahres-Berichte ins Deutsche übersetzt hatte, ist die erste Tendenz deutlich zu skizzieren. Wöhler, der Liebig auch zu einer angemessenen Diskussionsform aufforderte, stellte fest: „Zudem bin ich überzeugt, dass wenn du noch einmal Berzelius Aufsatz über Katalyse (im Jahresber.) aufmerksam durchliest, du finden wirst, dass er damit nicht mehr meint und will als wir auch, dass er nur eine Bezeichnung für eine Gruppe von Erscheinungen geben wollte, die uns bis jetzt unerklärlich sind und dass er so wenig wie wir an eine neue besondere Kraft glaubt" [17].

Liebig nahm diesen Hinweis nicht gerade dankend auf und antwortete: „Weisst du denn nicht, dass die Esel, welche in Deutschland Bücher schreiben, seine katalytische Kraft ohne zu prüfen, annehmen und unsern Kindern in den Kopf setzen werden ...? Gibst du nicht zu, dass die ganze Idee von der katalytischen Kraft falsch ist?" [18] Für die Anspannung, in der sich Liebig zu dieser Zeit möglicherweise befunden hat, und mit ihr für die Radikalität der Zurückweisung, nannte Wilhelm Ostwald späterhin einen möglichen Grund: Wegen seiner ,eben durchgemachten naturphilosophischen Kinderkrankheit“ hatte Liebig die „schädlichen Wirkungen“ von „Scheinerklärungen“ in „lebhaftester Erinnerung“ und reagierte daher heftig auf alles, was den Anschein einer „,neue(n) qualitas occulta, der eben überwundenen ,Lebenskraft" vergleichbar" hatte und ,in die Wissenschaft eingeführt werden sollte“ [19].

Die Beobachtungen, die zur Einführung des Katalysebegriffs geführt hatten, stellte Liebig keineswegs in Abrede, doch vertrat er zum Begriff auch späterhin eine dezidierte Meinung: „Noch heute können viele Chemiker Kollektivnamen, ähnlich dem Worte Phlogiston, für Vorgänge, von denen man vermutet, daß sie in eine Klasse gehören oder von derselben Ursache bedingt werden, nicht entbehren; aber anstatt hierzu Worte zu wählen, welche Dinge bezeichnen, wie dies bis Ende des 18. Jahrhunderts gewöhnlich war, bedienen wir uns seit Berthollet eigens für diesen Zweck erfundener ,Kräfte‘. So giebt es kaum etwas, was gegen die Regeln ächter Naturforschung mehr streitet, als die Erfindung und der Gebrauch des Wortes Katalyse oder katalytische Kraft; wir alle wissen, daß in diesem Worte keine Wahrheit liegt" [20]. Liebig selbst schlug stattdessen eine Erklärung des Phänomens vor, die im Sinn einer Übertragung der Bewegung von Atomen auf ein Nachbarsystem lautete: „Diese Ursache ist die Fähigkeit, welche ein in Zersetzung oder Verbindung, $d$.h. in einer chemischen Action begriffener Körper besitzt, in einem andern ihn berührenden Körper dieselbe Thätigkeit hervorzurufen oder ihn fähig zu machen, dieselbe Veränderung zu erleiden, die er selbst erfährt" [21]. Damit nahm Liebig zur ersten Theorie des Phänomens ausgerechnet eine Erklärung auf, die Georg Ernst Stahl in seiner ,Zymotechnia fundamentalis“ (1697; dt. 1734) unter alchemistischen Prämissen für die erste Theorie des Gärungsvorgangs entwickelt hatte.

Die zweite Tendenz in der Rezeption „katalytischer Kraft“" wurde, wie angedeutet, durch Julius Robert Mayer verkörpert. Ihm verdanken wir be- 
kanntlich das 1840 entdeckte Gesetz von der Erhaltung der Energie, womit er sich nach langen Auseinandersetzungen mit Helmholtz, Joule u. a. durchsetzen konnte. In seinen späteren Schriften führte ihn Berzelius' Entdeckung zu einer Differenzierung des Kraft- und Kausalitätsverständnisses: „, Katalytisch“ heißt eine Kraft, soferne sie mit der gedachten Wirkung in keinerlei Grössenbeziehung steht. Eine Lawine stürzt in das Thal; der Windstoss oder Flügelschlag des Vogels ist die ,katalytische Kraft', welche zum Sturze das Signal gibt und die ausgebreitete Zerstörung bewirkt. - Das ,katalytische' oder ,paralytische' dieser Kraft bezieht sich zu allernächst auf die Logik, oder das CausalGesetz“ [22]. In diesem Beitrag aus dem Jahr 1845 betonte Mayer allerdings auch, dass die Rede von ,katalytischer Kraft“ oder „katalytischer Wirkung“ dann unangemessen sei, wenn ,unter Kraft nur die, einer messbaren Wirkung proportionale messbare Ursache verstanden werden soll“" [23]. Die hier angesprochene Aussage ,Kleine Ursachen, große Wirkungen“ begegnet uns auch in der modernen Chaosforschung wieder, und zwar in Form des viel zitierten ,Schmetterlingseffekts‘ als Folge der Nichtlinearität gekoppelter Differentialgleichungen, ohne dass dafür die Gültigkeit des ,normalen“ Kausalitätsprinzips in Frage zu stellen wäre.

Ab 1851 setzte Mayer eine Definition von Kraft voraus, die ,nun bekanntlich so lautete: Kraft ist: Alles was eine Bewegung hervorbringt, oder hervorzubringen strebt, abändert oder abzuändern strebt (... die letzten eilf Wörter derselben (können) gestrichen werden (...), ohne dass dadurch der Sinn ein anderer wird)“ [24]. Vor diesem Hintergrund fasste er im 1876 erschienenen Aufsatz „Über Auslösung“, begrifflich differenziert, die Schlussfolgerungen zusammen, die er im Ausgang von der „katalytischen Kraft“ gewonnen hatte: „Sehr viele Naturprozesse gehen nur dann vor sich, wenn sie durch einen Anstoß eingeleitet werden, und dieser Vorgang ist es, welchen die neuere Wissenschaft ,die Auslösung' nennt. (...) Man könnte freilich bei der Auslösung statt Ursache und Wirkung auch Anstoß oder Veranlassung und Erfolg sagen“ [25]. Schließlich äußerte er auch folgende Vermutung: „Die Auslösungen spielen nicht nur in der anorganischen Natur, sondern auch in der lebenden Welt, und namentlich also in der Physiologie und Psychologie, eine große und wichtige Rolle“, und nannte, passend zum Vorbild der Knallgas-Reaktion, als literarischen Beleg dafür: „Freude, schöner Götterfunken“ [26].

Im übrigen war die zweite Hälfte des 19. Jahrhunderts bezüglich des Begriffs „Katalyse“ durch eine bemerkenswerte Zwiespältigkeit gekennzeichnet: Während im Zuge der aufkommenden Industrialisierung katalytische Prozesse in der Technik zunehmend an Bedeutung gewannen, trat das Interesse nach einer wissenschaftlichen Erklärung des Phänomens weitgehend in den Hintergrund - letzteres wohl nicht zuletzt gefördert durch die scharfe Opposition Liebigs. Eine Neubelebung und Wende in der Diskussion erfolgte erst bei Wilhelm Ostwald.

Als ersten Hinweis auf die von ihm vorgenommene Verknüpfung von Katalyse mit dem neuen Gebiet der chemischen Reaktionskinetik kann man wohl 
folgende 1894 publizierte Definition betrachten: „Katalyse ist die Beschleunigung eines langsam verlaufenden chemischen Vorgangs durch die Gegenwart eines fremden Stoffes“ [27]. Den Kenntnisstand seiner Zeit zum Thema „Katalyse" schilderte Ostwald ausführlich und kenntnisreich in dem 1897/1898 erschienenen Beitrag „Aeltere Lehre von den Berührungswirkungen“. Bemerkenswerterweise handelte es sich bei dieser chemiehistorischen Arbeit um eine bei der Philosophischen Fakultät der Universität Leipzig eingereichte „dissertatio“, deren Autor, bereits mehrfacher Doktor, dort ordentlicher Professor der Chemie und zu dieser Zeit Dekan war! Am Schluß der Abhandlung steht zu lesen: „... das Erlahmen des Interesses (wird) wohl auch dem Umstande zuzuschreiben sein, dass eine einigermassen befriedigende Theorie dieser Erscheinungen sich nicht ausfindig machen liess. (...) Erst in neuester Zeit beginnt sich eine neue Entwicklung des alten Problems geltend $\mathrm{zu}$ machen. Durch die fundamentale Wendung, nach der die katalytischen Erscheinungen nicht mehr als Vorgänge besonderer Art, sondern als Beschleunigungen, bez. Verzögerungen vorhandener Vorgänge aufgefasst werden, sind die hier zu stellenden Fragen auf eine bestimmtere Form gebracht worden, und es steht zu hoffen, dass die bestimmtere Fragestellung auch bestimmtere Antworten ermöglichen wird" [28].

Bevor Ostwald in der Folgezeit versuchte, zu diesen ,,bestimmteren Antworten“ zu gelangen, setzte er sich zunächst einmal mit den bis dato entwickelten Vorstellungen auseinander. So führte er in der genannten Dissertation auch die Argumente Liebigs an, meinte aber, diese bräuchten nicht einzeln diskutiert zu werden, ,zumal sich verschiedene von ihnen später als irrig erwiesen haben“ [29]. Für Liebigs oben genannte theoretische Erklärung hieß das: „Bereits auf seine Freunde scheint diese Ansicht nicht überzeugend gewirkt zu haben, denn in einer Nachschrift giebt Liebig an, sie hätten gemeint, dass sich nicht einsehen lasse, wie und auf welche Weise diese Ursache die Erscheinung bewirke“ [30]. Und da Liebigs Hypothese zu Ostwalds Zeit bereits allgemein als erledigt galt, beschäftigte auch er sich nicht mehr weiter damit.

Anders stellte sich die Situation in bezug auf Julius Robert Mayer dar. Hier trennte Ostwald zur begrifflichen und sachlichen Klarstellung „Auslösungserscheinungen“ gänzlich vom Bereich „,katalytischer Vorgänge“: „Die Explosion eines Knallgasgemisches durch den elektrischen Funken soll nicht als eine katalytische Erscheinung aufgefasst werden, wohl aber die langsame Verbindung des Knallgases durch die Wirkung des feinstzertheilten Platins“ [31]. In diesem Sinn hob er 1894 die „Aenderung des zeitlichen Maasstabes“ als wesentliches Moment katalytischer Vorgänge hervor: „Als katalytische Erscheinung definire ich den Vorgang, dass eine für sich in einer bestimmten Zeit verlaufende chemische Reaction durch die Gegenwart eines fremden Stoffes, der am Ende der Reaction in demselben Zustande ist, wie am Anfange, eine Aenderung seines zeitlichen Verlaufes erfährt“ [32]. Den Maßstab für den zeitlichen Verlauf gaben mechanische Vorgänge, die mit „Bewegungsenergie“ verknüpft, im Sinn der Energieerhaltung kalkulierbar und insofern ,zeitlich bestimmt“ sind [33]. 
Das Überschreiten der „mechanistische(n) Hypothese“ sah Ostwald vor allem im Bereich der „,natürlichen Vorgänge“, so wirkten insbesondere Fermente als „specielle() Katalysatoren“, wobei „deren Wirkungen sich allerdings in den meisten Fällen unter so verwickelten Bedingungen geltend machen, dass der Nachweis der hier obwaltenden Gesetzmässigkeiten noch lange Zeit und viele Arbeit erfordern wird" [34].

Darüber hinaus fand aber das im Rahmen der Auslösungtheorie von Mayer auf psychische Vorgänge erweiterte Spektrum katalytischer Vorgänge auch bei Wilhelm Ostwald Widerhall: Auf der Grundlage der Entgegensetzung einer empfundenen Willensfreiheit qua Freiheit der Wahl - eine „Empfindung ..., die jedem Menschen eigen ist“ - und der „Folgerung aus der mechanistischen Weltanschauung ..., nach welcher der ganze Verlauf der Welt, nachdem die Anfangsbedingungen gegeben sind, unabänderlich in einer dadurch endgültig bestimmten Weise stattfinden muß“, entwarf er ein Konzept, das auch seinen eigenen ganzheitlichen Ansprüchen gerecht wurde: „Sämmtliche geistigen Vorgänge dürfen wir als unlösbar mit materiellen, insbesondere chemischen verbunden betrachten, und der Verlauf der ersteren wird durch dieselben Ursachen beeinflusst werden, welche auf die letzteren wirken“ [35].

In Analogie zur Auffassung von Katalyse in chemischer Hinsicht implizierte das auch die „Möglichkeit, das Zeitmaass der psychischen Vorgänge zu regeln, wenn auch das Eintreten derselben naturgesetzlich, d. h. energetisch bestimmt ist“", und so den Determinismus der Bestimmung des Willens maßvoll zu durchbrechen: „Wir sind nicht frei darin, dass wir z. B. beim Anblick eines erwünschten Dinges es nicht begehrten, wohl aber sind wir frei darin, dass wir die neben dem Begehren auftretenden Gedankenreihen, welche etwa uns die Besitzergreifung als ein Unrecht erscheinen lassen, schneller oder langsamer, und demgemäss wirksamer oder weniger wirksam stattfinden lassen“ [36]. Die Frage, welche Reihe letzthin wirksam ist, sollte sich nach der Intensität, d.h. hier der Beschleunigung des entsprechenden ,,psycho-physischen Vorgang(s)“, entscheiden; dieses Muster galt Ostwald für alle „geistigen Vorgänge“, sofern sie ,zu einer Handlung“ führten [37].

Seine begrenzte „Kenntniss der Psychologie“ voraussetzend, formulierte Ostwald vorsichtig als Fazit: ,,Verfügt daher der Mensch über ein Mittel, katalytische Wirkungen bei dem Ablauf der mit den geistigen Vorgängen verbundenen chemischen zur Geltung zu bringen, so hat er dadurch die Möglichkeit, diese geistigen Vorgänge ... zu beschleunigen oder zu verlangsamen“ [38]. Das Problem der Willensfreiheit, spätestens seit Ostwald auch in der Naturwissenschaft diskutiert, stellt heutzutage noch eines der größten (und vielleicht niemals lösbaren) Rätsel dar, dessen philosophische Prägung auch weiterhin eine Rolle spielt. So schlägt beispielsweise der Hirnforscher Wolf Singer vor, „,dass wir die Erfahrung, ein freies, selbstbestimmtes Ich zu sein, aus der Spiegelung unseres Selbst im jeweils andern gewinnen“, und meint aber auch: „Wir wissen heute, dass wir nicht wissen können, wo uns die Grenzen des Wißbaren gezogen sind" [39]. 
Nachdem Ostwald sich bereits am Anfang seiner Forscherlaufbahn mit Fragen zur Kinetik chemischer Reaktionen beschäftigt hatte, begann er etwa zeitgleich mit der Abfassung der oben genannten Dissertation und dem Einzug in sein neues Leipziger Institutsgebäude, sich 1897/98 verstärkt dem praktischen Nutzen der Katalyse zuzuwenden. Zur Nutzanwendung gelangte u. a. das von ihm in dieser Periode entwickelte Ostwald-Verfahren zur Oxidation von Ammoniak für die Produktion von Salpetersäure, das noch immer großtechnischen Einsatz findet [40]. Als Ergebnis seiner Überlegungen zu „Katalyse“ präsentierte er bei einem Vortrag am 26. September 1901 anlässlich der 73. Versammlung der Gesellschaft Deutscher Naturforscher und Ärzte seine endgültige Definition: „Ein Katalysator ist jeder Stoff, der, ohne im Endprodukt einer chemischen Reaktion zu erscheinen, ihre Geschwindigkeit verändert" [41].

Einen Hinweis auf die erstmalige Erkenntnis von Katalyse als kinetisches Phänomen gab er in der Erläuterung seiner 1887 durchgeführten Untersuchungen zur Oxidation von Jodwasserstoff durch Bromsäure, wie er in seiner Nobelpreis-Rede vortrug: „So wurde ich unwiderstehlich zu der Auffassung gedrängt, dass das Wesen der Katalyse nicht in der Hervorbringung einer Reaktion zu suchen ist, sondern in ihrer Beschleunigung", und gestand aber auch ein: „Ich würde der Pflicht der Aufrichtigkeit ... zuwider handeln, wenn ich unterlassen würde, zu bemerken, daß mir selbst damals dieser Fortschritt keineswegs besonders imponierte. (...) Erst ... etwas später empfand ich auch das Bedürfnis, die Stagnation zu beseitigen, in welche die Erforschung der katalytischen Erscheinungen ... geraten war. Ich erinnerte mich der naiven Zeichnungen, die ein namhafter Forscher in jener Zeit veröffentlicht hatte, um sich den katalytischen Einfluß von gestoßenem Glase auf die Bestandteile des Knallgases bei mäßiger Erwärmung zu ,veranschaulichen'; es war dargestellt worden, wie die scharfen Kanten der Glassplitter die Molekeln der Gase in Atome zerschnitten, die sich hernach ungehindert verbinden konnten" [42].

Eine derartige Vorstellung musste Ostwald allein deshalb ein Gräuel sein, weil er in dieser Zeit zu den Gegnern der von Boltzmann angeführten Anhänger der atomistischen Vorstellung von der Materie zählte und sich erst später vom Saulus zum Paulus wandelte. Ironischerweise ist unser heutiges Bild über die atomaren Einzelschritte der katalytischen Knallgas-Reaktion gar nicht so verschieden von den Vorstellungen, die Ostwald hier verspottete, was weiter unten näher erläutert wird. Auf der anderen Seite macht dieses Zitat deutlich, welche Revolution Ostwalds Definition der Katalyse darstellte, wie auch 1909 in der Verleihung des Nobelpreises zum Ausdruck gebracht wurde: ,als Anerkennung für seine Arbeiten über Katalyse und seine dafür grundlegenden Untersuchungen über chemische Gleichgewichtsverhältnisse und Reaktionsgeschwindigkeiten“ [43].

Den Begriff „Katalyse“ hielt Ostwald für äußerst zutreffend, und so nannte er rückblickend in seiner Rede anlässlich der Übergabe des Preises Berzelius einen „Meister der chemischen Begriffsbildung“, der auch im Fall von „Katalyse“ die Fähigkeit bewiesen habe, den Begriff „,so rein und vollkom- 
men“ herzustellen, ,als es unter den gegebenen Bedingungen nur irgend möglich war" [44]. Angesichts seiner eigenen Leistung stellte er am Schluss der Rede fest: „... die Antwort auf die Frage, welche Arten Reaktionen katalysiert werden, und in welchen Beziehungen die chemische Beschaffenheit des Katalysators zu der der reagierenden Stoffe steht, ist einstweilen noch kaum beantwortbar. Was wir hierüber sagen können, ist im Falle der auf Zwischenreaktionen beruhenden Katalyse, daß die Stoffe in solchen Beziehungen stehen müssen, daß solche mögliche(!) Zwischenreaktionen vorhanden sind, die insgesamt viel schneller verlaufen, als die unmittelbare oder Hauptreaktion" [45]. Damit wurde, wenn auch auf etwas verschlungene Weise, die Hypothese formuliert, dass der Katalysator mit den an der Reaktion beteiligten Stoffen Zwischenverbindungen eingehen kann, über die insgesamt der Weg zum Produkt schneller durchlaufen wird. Ostwald fuhr fort: „Hieraus erkennt man ..., daß, solange die Frage nach der allgemeinen Vorausberechnung einer chemischen Reaktionsgeschwindigkeit ... noch nicht gelöst ist, eine ausreichende Antwort auf die katalytische Frage nicht gegeben werden kann“ [46]. Die Beantwortung dieser Fragen konnte erst in jüngster Zeit in Angriff genommen werden. Ostwald selbst, der bereits 1906 seinen Lehrstuhl aufgegeben hatte, wandte sich in der Folgezeit einer Vielzahl ganz anderer Probleme zu.

Schon zu Ostwalds Zeit erfolgte die Unterteilung in homogene, heterogene und biologische (enzymatische) Katalyse. Während die heterogene (Grenzflächen-) Katalyse insbesondere seit Beginn des 20. Jahrhunderts einen wahren Siegeszug in der chemischen Technik antrat, setzte das theoretische Verständnis dieses Phänomens erst verzögert ein und ist in erster Linie mit dem Namen Irving Langmuir verbunden. Dieser erkannte u. a. die Schlüsselrolle der Ausbildung chemischer Bindungen zwischen den an der Reaktion beteiligten Molekülen und den unabgesättigten Valenzen der Oberflächenatome (Chemisorption). Damit können auch die von Ostwald postulierten ,Zwischenreaktionen' identifiziert werden. Langmuir schlug ferner vor, zum Studium der Elementarschritte anstelle der schlecht definierten Oberflächen ,realer' Katalysatoren als Modellsystem wohldefinierte Einkristall-Oberflächen zu verwenden [47]. Es dauerte noch rund ein halbes Jahrhundert, bis dieses Konzept als sog. ,surface science approach“ experimentell realisiert werden konnte. Parallel dazu erfolgte die Weiterentwicklung der theoretischen Werkzeuge zur quantenmechanischen Behandlung der Chemisorptionsbindung, so dass heute das von Ostwald gesteckte Ziel zumindest in einfachen Fällen als erreicht betrachtet werden kann.

Dies soll im folgenden kurz anhand der eingangs erwähnten von Döbereiner entdeckten Platin-katalysierten Knallgasreaktion erläutert werden [48]: Beide Reaktionspartner werden bei Wechselwirkung mit einer Platin-Oberfläche unter Bindungsbruch adsorbiert, d. h. $\mathrm{O}_{2} \rightarrow 2 \mathrm{O}_{\text {ad }}$ und $\mathrm{H}_{2} \rightarrow 2 \mathrm{H}_{\text {ad }}$. Die dabei freigesetzte Chemisorptionsenergie ermöglicht die Spaltung der Moleküle ohne nennenswerten Aufwand und daher mit großer Wahrscheinlichkeit und tritt damit anstelle des oben zitierten, von Ostwald verspotteten ,Zerschnei- 
dens'. Die chemisorbierten H-Atome besitzen eine extrem hohe Beweglichkeit auf der Oberfläche und können durch Wechselwirkung mit $\mathrm{O}_{a d}$ zur Bildung von adsorbierten $\mathrm{OH}_{a d}$-Spezies führen, die mit $\mathrm{H}_{\text {ad }} \mathrm{zu} \mathrm{H}_{2} \mathrm{O}_{\text {ad }}$ weiterreagieren, wobei das gebildete Wasser in einem letzten Teilschritt in die Gasphase übertritt (desorbiert). Dieses einfache Bild wird aber dadurch komplizierter, als beobachtet wurde, dass der Mechanismus der Reaktion durch die Anwesenheit von $\mathrm{H}_{2} \mathrm{O}$ auf der Oberfläche beeinflusst wird. Es stellte sich heraus, dass $\mathrm{OH}_{a d}$ auch über die Reaktion des Produkts $\mathrm{H}_{2} \mathrm{O}_{a d}$ mit $\mathrm{O}_{\text {ad }}$ gebildet werden kann. Dieser Schritt verläuft sogar schneller (d.h. mit geringerer Aktivierungsenergie) als die direkte Rekombination $\mathrm{O}_{\mathrm{ad}}+\mathrm{H}_{\mathrm{ad}}$ und bedeutet, dass die Reaktion autokatalytisch verläuft, d.h. durch das Reaktionsprodukt selbst beschleunigt wird. Nachfolgende quantenmechanische Rechnungen zu den Bindungsstärken und Aktivierungsbarrieren der Einzelschritte [49] bestätigten diesen experimentellen Befund, der im wesentlichen auf atomar aufgelösten Abbildungen der Oberflächen und Ermittlung der Schwingungsfrequenzen der adsorbierten Spezies beruht. Allerdings bedürfen auch hier gewisse Details noch einer weiteren Klärung.

Der Befund, dass Wasser bei einer Oxidationsreaktion eine wesentliche Rolle spielt, ist keineswegs neu, sondern führt zurück auf eine Veröffentlichung, in der das Prinzip der Katalyse schon lange vor Berzelius angesprochen wurde: 1794 hatte Mrs. Elizabeth Fulhame, die Frau eines englischen Arztes, ein Buch mit dem Titel „An essay on combustion, with a view to a new art of dying and painting“ publiziert. Darin hielt sie fest, ,that water is the only source of oxygen, which oxygenates combustible bodies" [50]. Und an anderer Stelle: ,... the carbone attracts the oxygen of the water, and forms carbonic acid, while the hydrogen of the water unites with oxygen of the vital air, and forms a new quantity of water equal to that decomposed" [51]. In der Formelsprache des Chemikers lautet dies: $\mathrm{C}+2 \mathrm{H}_{2} \mathrm{O} \rightarrow \mathrm{CO}_{2}+4 \mathrm{H}$ und $4 \mathrm{H}+\mathrm{O}_{2} \rightarrow 2 \mathrm{H}_{2} \mathrm{O}$, d.h. $\mathrm{H}_{2} \mathrm{O}$ wirkt hiernach als Katalysator. Mrs. Fulhame's Buch erschien 1798 in einer deutschen Übersetzung sowie 1810 in den USA und wurde vereinzelt rezensiert, versank aber ansonsten in der Versenkung [52]. Im Jahr 1903, also nicht lange, nachdem Ostwald die allgemeingültige Definition des Begriffs „Katalyse“ aufgestellt hatte, erschien ein Kommentar von J. W. Mellor, der unter dem Titel „History of the water problem (Mrs. Fulhame's theory of catalysis)" einmal mehr die Bedeutung von Ostwalds Leistung reflektiert [53].

\section{References}

1. J. Schiff (Hrsg.), Briefwechsel zwischen Goethe und Johann Wolfgang Döbereiner (1810-1830) (1914) 78.

2. dazu A. Mittasch und E. Theis, Von Davy bis Deacon. Ein halbes Jahrhundert Grenzflächenkatalyse (1932), 31-39; zit. Döbereiner 34.

3. E. Mitscherlich, Annalen der Physik und Chemie 31 (1934) 273-282, zit. 281. 
4. H. Kopp, Die Alchemie in älterer und neuerer Zeit (Heidelberg 1886. ND 1962), 9.

5. in Ref. [4], $87 \mathrm{f}$.

6. C. Priesner und K. Figala (Hrsg.), Alchemie. Lexikon einer hermetischen Wissenschaft (1998), Art. „Lapis philosophorum“, 219.

7. W. Ostwald, Das große Elixier. Die Wissenschaftslehre (1920), $7 \mathrm{f}$.

8. J. Liebig, Chemische Briefe (4., umgearbeitete und vermehrte Aufl. 1859), 67; 70.

9. J. J. Berzelius, Einige Ideen über eine bei der Bildung organischer Verbindungen in der lebenden Natur wirksame, aber bisher nicht bemerkte Kraft, Jahres-Bericht über die Fortschritte der physischen Wissenschaften. Eingereicht durch die schwedische Akademie der Wissenschaften, den 31. März 1835. Aus dem Schwedischen übersetzt von F. Wöhler, Fünfzehnter Jahrgang (1836), 237-245, zit. 243.

10. in Ref. [9], $242 \mathrm{f}$.

11. in Ref. [9], $243 \mathrm{f}$.

12. J. Carrière (Hrsg.), Berzelius und Liebig. Ihre Briefe von 1831-1845 mit erläuternden Einschaltungen aus den gleichzeitigen Briefen von Liebig und Wöhler (1893), 107.

13. in Ref. [9], 234.

14. in Ref. [9], 245.

15. P. Walden, Z. Angew. Chem. 40 (1939) 12.

16. J. J. Berzelius, Lehrbuch der Chemie I (5. Auflage, deutsch 1843), 110.

17. in Ref. [12], Brief Wöhlers vom 30. Mai 1837, 132.

18. in Ref. [12], Brief Liebigs vom 2. Juni 1837, 133.

19. W. Ostwald, Chemische Theorie der Willensfreiheit, Ber. Vh. der Kgl. Sächs. Ges. der Wiss. zu Leipzig. Math.-phys. Kl. 46 (1894) 334-343, zit. 337.

20. in Ref. [8], 74.

21. J. Liebig, Lieb. Ann. 30 (1839) 250-287; Nachtrag 363-368, zit. 262.

22. J. R. Mayer: Die organische Bewegung in ihrem Zusammenhange mit dem Stoffwelchsel (1845), in: ders.: Mechanik der Wärme. Sämtliche Schriften, hrsg. von H. P. Münzenmayer (1978), 41-154, zit. 122, Anm.

23. in Ref. [22], 122.

24. J. R. Mayer, Bemerkungen über das mechanische Aequivalent der Wärme, in Ref. [22], 253-308, zit. 276.

25. J. R. Mayer, Ueber Auslösung (1876), in Ref. [22], 411-416, zit. 413.

26. in Ref. [25], 414.

27. W. Ostwald, Z. Phys. Chem. 15 (1894) 706.

28. W. Ostwald, Aeltere Lehre von den Berührungswirkungen, Leipzig (1897/98), 44.

29. in Ref. [28], 41.

30. in Ref. [28], 42.

31.-32. in Ref. [19], 338.

33. in Ref. [19], 336.

34. in Ref. [19], 336; 341.

35.-38. in Ref. [19], 342.

39. W. Singer, Ignorabimus? - Ignoramus. Jahrbuch 2000 der Berlin-Brandenburgischen Akademie der Wissenschaften (2002), 115-124, zit. 124.

40. dazu z. B. A. Mittasch, Salpetersäure aus Ammoniak (1953).

41. W. Ostwald, Phys. Z. 3 (1902), 313-322; zit. nach Ostwald's Klassiker Nr. 200, hrsg. von G. Bredig (1923), 29.

42. W. Ostwald, Über Katalyse. Rede gehalten am 12. Dezember 1909 beim Empfang des Nobelpreises für Chemie. Ann. der Naturphilosophie 9 (1910) 1-25, zit. 15; 16.

43. aus dem Text der Nobelpreisurkunde.

44. in Ref. [42], $4 ; 6$.

45.-46. in Ref. [42], 25.

47. I. Langmuir, Trans. Faraday Soc. 17 (1922) 607. 
48. dazu S. Völkening, K. Bedürftig, K. Jacobi, J. Wintterlin und G. Ertl, Phys. Rev. Lett. 83 (1999) 2672-2675.

49. A. Michaelides und P. Hu, J. Am. Chem. Soc. 123 (2001) 4235-4242.

50. E. Fulhame: An essay on combustion, with a view to a new art of dying and painting, wherein the phlogistic and antiphlogistic hypotheses are proved erroneous, Cooper and Johnson, London (1794).

51. in Ref. [50].

52. D. A. Davenport und K. M. Ireland, Bull. Hist. Chem. 5 (1984) 37-42.

53. J. W. Mellor, J. Phys. Chem. 7 (1903) 557-567. 